\title{
AND SOLAR'SYMPATHETIC' FLARES
}

\author{
M. RODONÒ \\ Catania Astrophysical Observatory and Institute of Mathematics, \\ University of Messina, Italy
}

\begin{abstract}
About $50 \%$ of the flare events observed on red dwarfs are at least double-peaked. As the majority of flare stars are members of double or multiple systems, the possibility that time-overlapping flares originate quasi-simultaneously on the individual components is discussed.

Assuming a poissonian occurrence of flares in both components, the expected probability of observing double-peaked flares is lower than $1 \%$ for the most active binary systems.

However, from photometric observations of the double flare star EQ Peg (BD $+19^{\circ} 5116 \mathrm{AB}$ ) carried out by the author with an area scanner (the components' angular separation is $3.7^{\prime \prime}$ ) about $20 \%$ of the observed flares have been found to be double-peaked flares resulting from separate flares, one in each component. A direct flare triggering of the following flare by the preceding one can be ruled out since the light travel-time between the two components is $3.5 \mathrm{~h}$, while the observed time delay between the flare peaks is about $10 \mathrm{~min}$. Moreover, the proximity effect does not seem to play an important triggering role.

It is concluded that, although the analogy with solar 'sympathetic' flares is not always applicable, it is the most promising framework within which the majority of double-peaked flare events on red dwarfs must be interpreted.
\end{abstract}

The results here presented are included in the paper 'Flare Activity on Binary Systems' submitted to Astronomy and Astrophysics. 